\title{
Body Mass Index and Clinical Outcomes in Large Vessel Occlusion Acute Ischemic Stroke after Endovascular Therapy
}

\author{
Mehdi Bouslama Hilarie J. Perez Clara M. Barreira Diogo C. Haussen \\ Jonathan A. Grossberg Samir R. Belagaje Nicolas A. Bianchi \\ Aaron M. Anderson Michael R. Frankel Raul G. Nogueira \\ Marcus Stroke and Neuroscience Center, Grady Memorial Hospital, Atlanta, GA, USA; \\ Departments of Neurology, Radiology, and Neurosurgery, Emory University School of \\ Medicine, Atlanta, GA, USA
}

\section{Keywords}

Acute stroke $\cdot$ Cerebral ischemia $\cdot$ Thrombectomy $\cdot$ Obesity $\cdot$ Body mass index

\begin{abstract}
Background and Purpose: Several reports have described lower mortality rates in overweight or obese patients as compared to normal weight ones. In the past decade, several studies have investigated the phenomenon, commonly known as the obesity paradox, with mixed results thus far. We sought to determine whether outcomes differ between patients with large vessel occlusion strokes (LVOS) after endovascular therapy (ET) according to their body mass index (BMI). Methods: We reviewed our prospectively collected endovascular database at a tertiary care academic institution. All patients that underwent ET for acute LVOS were categorized according to their BMI into 4 groups: (1) underweight (BMI <18.5), (2) normal weight (BMI = 18.5-25), (3) overweight (BMI = 25-30), and (4) obese (BMI >30). Baseline characteristics, procedural radiological as well as outcome parameters where compared. Results: A total of 926 patients qualified for the study, of which 20 (2.2\%) were underweight, $253(27.3 \%)$ had a normal weight, 315 (34\%) were overweight, and 338 (36.5\%) were obese. When compared with normal weight (reference), overweight patients were younger, had higher rates of dyslipidemia and diabetes and higher glucose levels, while obese patients were younger, less often smokers, and had higher rates of hypertension and diabetes and higher glucose levels. Other baseline and procedural characteristics were comparable. The rates of successful reperfusion (modified
\end{abstract}


treatment in cerebral ischemia, 2b-3), parenchymal hematomas, 90-day good clinical outcomes (modified Rankin scale, $0-2$ ), and 90-day mortality were comparable between groups. On multivariate analysis, BMI was not associated with good outcomes nor mortality. Conclusion: In patients treated with mechanical thrombectomy, BMI is not associated with outcomes. However, patients who are overweight or obese have more comorbidities and a higher stroke risk and, thus, should strive for a normal weight.

(c) 2019 S. Karger AG, Base

\section{Introduction}

Over the past few decades, the prevalence of obesity in the US has increased from 13 to $35.5 \%$ [1]. Obesity is known to be associated with increased mortality and morbidity in the general population and is an established risk factor leading to stroke [2-4].

However, its influence on stroke outcomes remains under debate. In fact, several studies have shown a better clinical poststroke outcome in obese patients than in those of normal weight, a phenomenon commonly described as the "obesity paradox," such as better functional outcomes, lower re-hospitalization rates, and lower rates of stroke reoccurrence [5-9]. Nevertheless, reports have been widely conflicting, and explanations currently remain ambiguous [10-14].

Specifically regarding large vessel occlusion strokes, the potential association of body mass index (BMI) with post-stroke clinical outcomes for patients receiving endovascular therapy (ET) is relatively unexplored. As an "obesity paradox" could inform post-stroke clinical care and secondary stroke prevention measures, the need for further investigation is warranted.

Thus, we assessed the association between BMI and clinical outcomes in patients with large vessel occlusion strokes undergoing ET.

\section{Methods}

We reviewed our prospectively collected Grady Endovascular Stroke Therapy Outcomes Registry (GESTOR) between September 1, 2010, and March 30, 2016, to identify patients with large vessel occlusion strokes who underwent ET with documented BMI.

Patients were then categorized into 4 groups: (1) underweight $(\mathrm{BMI}<18.5),(2)$ normal weight $(\mathrm{BMI}=$ 18.5-25), (3) overweight (BMI = 25-30), and (4) obese (BMI >30). Baseline characteristics and demographics as well as procedural parameters were collected and compared.

The primary outcome measure was the overall degree of disability as measured by the modified Rankin scale (mRS) 90 days after stroke. Secondary endpoints included the rates of good outcomes (defined as 90-day mRS 0-2) and successful reperfusion as defined by a modified treatment in cerebral ischemia (mTICI) score of $2 b-3$. Safety endpoints included the rates of any parenchymal hematoma, as per the European Cooperative Acute Stroke Study (ECASS) criteria, and 90-day mortality.

This study was approved by the Emory University institutional review board.

Imaging Protocol/Computed Tomography Perfusion Parameters/Final Infarct Volume Calculation

All patients underwent an institutional imaging protocol, including non-contrast computed tomography (NCCT) \pm CT angiography, and CT perfusion. Imaging acquisition parameters were the same for all patients included in the study. CT perfusion was evaluated with a fully automated software environment (RAPID, version 4.5.0, iSchemaView, Menlo Park, CA, USA). The ischemic tissue volume (ischemic core) was defined by a voxel relative cerebral blood flow of $<30 \%$ of the normal tissues. The total hypoperfused volume was defined by $>6 \mathrm{~s}$ delay in the time-to-maximum of the tissue residue function and a penumbral volume of at-risk tissue defined by the difference between total hypoperfused and ischemic core tissue estimates [14]. 
Follow-up imaging included NCCT or magnetic resonance imaging (MRI) within 5 days of the treatment. For final infarct volume (FIV) calculation purposes, diffusion-weighted imaging was preferentially utilized if MRI was obtained within the first $72 \mathrm{~h}$ of the stroke, and fluid-attenuated inversion recovery was used if MRI was performed within 3-5 days. Edema-producing sulcal effacement was not excluded. Hemorrhagic transformation was incorporated in the FIV whenever present. FIV was measured following export of raw DICOM data to the Fiji release of the ImageJ software platform using a standardized, semi-automated approach (http://imagej.nih.gov).

\section{Statistical Analysis}

The Shapiro-Wilk test was used to assess the normality of the variables. Continuous variables were reported as means \pm standard deviations if normally distributed or medians (interquartile ranges) if nonparametric. Categorical variables were reported as proportions. Between groups, comparisons for continuous/ ordinal variables were made with the Student $t$ test, Mann-Whitney U test, and ANOVA, as appropriate. Categorical variables were compared by the $\chi^{2}$ test or Fisher exact test, as appropriate. Multivariate logistic regression analyses for predictors of good outcomes were performed for variables at the 0.1 level of significance on univariate analysis. Significance was set at $p<0.05$, and all $p$ values were two-sided.

Statistical analysis was performed using IBM ${ }^{\circledR}$ SPSS $^{\circledR}$ Statistics $23\left(\right.$ IBM $^{\circledR}$, Armonk, NY, USA) and SAS ${ }^{\circledR}$ University Edition (SAS Institute, Cary, NC, USA).

\section{Results}

During the study period, 971 patients underwent ET, of which 45 were missing BMI data and, thus, were excluded. A total of 926 patients underwent primary analysis, of which 20 $(2.2 \%)$ were underweight, $253(27.3 \%)$ had a normal weight, 315 (34\%) were overweight, and $338(36.5 \%)$ were obese.

When compared with normal weight (reference), overweight patients were younger (65 [56-76] vs. 70 [56.5-80] years, $p<0.03$ ), had higher rates of dyslipidemia (38.9 vs. $29.6 \%$, $p=0.03$ ) and diabetes ( 23.6 vs. $15.8 \%, p=0.03$ ), as well as higher glucose levels (123 [101145 ] vs. 116.5 [98-136] mg/dL, $p<0.01$ ). Obese patients were younger (60 [51.5-71.5] vs. 70 [56.5-80] years, $p<0.01$ ), less often smokers ( 14.6 vs. $25.3 \%, p<0.01$ ), had higher rates of hypertension (77.7 vs. $67.2 \%, p<0.01$ ) and diabetes ( 36.5 vs. $15.8 \%, p<0.01$ ), as well as higher glucose levels (124 [105-161] vs. 116.5 [98-136] mg/dL, $p<0.01)$. Other baseline characteristics, including National Institutes of Health Stroke Scale (NIHSS) and Alberta Stroke Program Early CT (ASPECT) scores as procedural characteristics, were comparable between groups (Table 1).

In terms of outcomes, rates of successful reperfusion (mTICI, 2b-3), parenchymal hematomas, 90-day good clinical outcomes (mRS, 0-2), and 90-day mortality were comparable across all BMI categories (Table 2).

On multivariate analysis, BMI was not associated with 90-day good outcomes nor mortality whether as a continuous variable or as a categorical variable comparing groups with normal weight patients (reference) 2 by 2 (Table 3 ).

\section{Discussion}

Obesity is a well-established cerebrovascular risk factor [4, 15]. In fact, every 1-unit increase in BMI results in an approximately 5\% increase in the risk for ischemic stroke [4]. Obese patients are also more likely to have hypercholesterolemia, hypertension, diabetes, and obstructive sleep apnea, which are risk factors for stroke and are also associated with worse post-stroke outcomes [4]. 


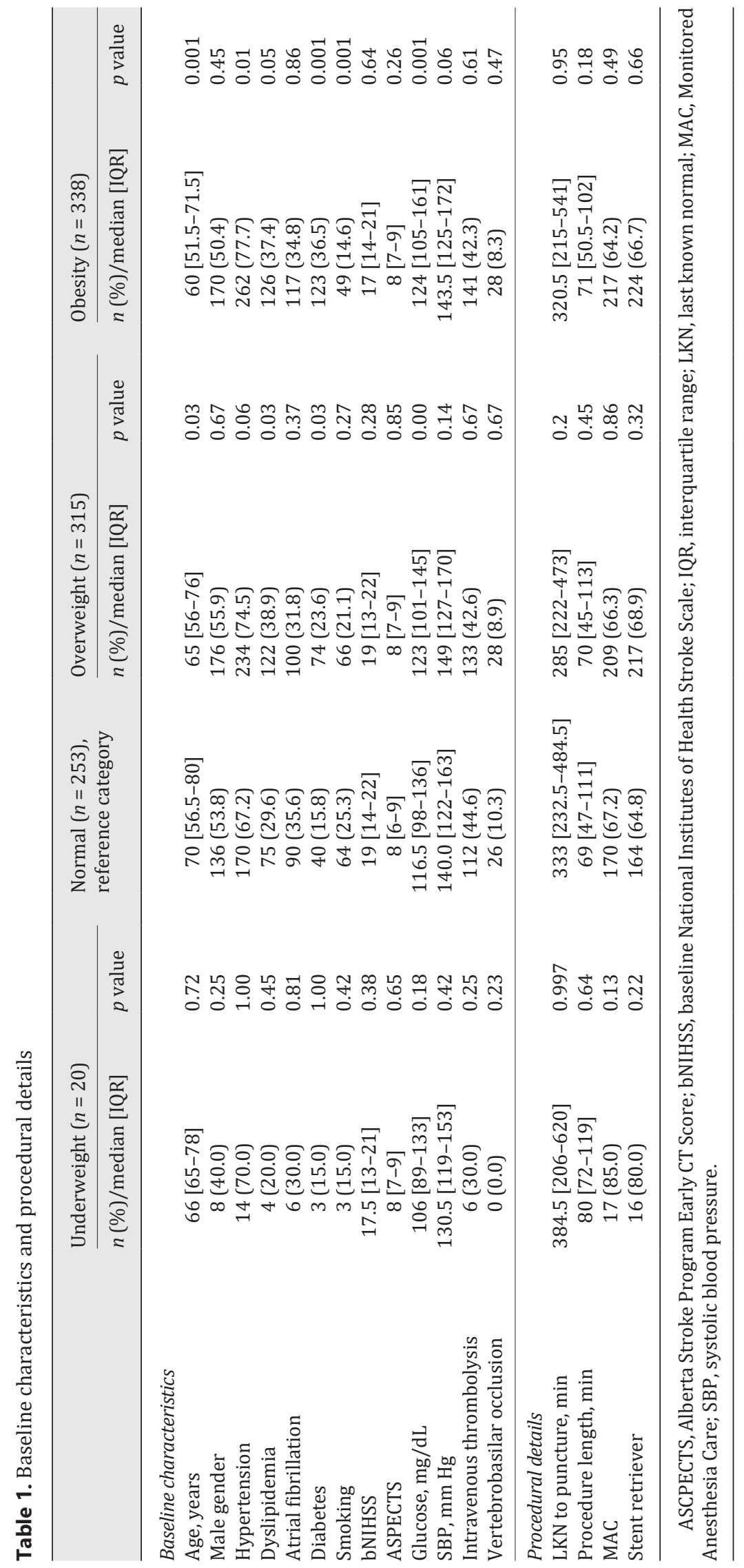


Table 2. Outcome measures

\begin{tabular}{|c|c|c|c|c|c|c|c|}
\hline & \multicolumn{2}{|c|}{ Underweight $(n=20)$} & \multirow{2}{*}{$\begin{array}{l}\text { Normal } \\
(n=253), \\
\text { reference } \\
\text { category }\end{array}$} & \multicolumn{2}{|c|}{ Overweight $(n=315)$} & \multicolumn{2}{|c|}{ Obesity $(n=338)$} \\
\hline & $\begin{array}{l}n(\%) / \\
\text { median [IQR] }\end{array}$ & $\begin{array}{l}p \\
\text { value }\end{array}$ & & $\begin{array}{l}n(\%) / \\
\text { median [IQR] }\end{array}$ & $\begin{array}{l}p \\
\text { value }\end{array}$ & $\begin{array}{l}n(\%) / \\
\text { median [IQR] }\end{array}$ & $p$ value \\
\hline mTICI $2 b-3$ & $18(90.0)$ & 1.00 & $228(90.5)$ & $278(88.3)$ & 0.42 & $293(87.5)$ & 0.29 \\
\hline mTICI 3 & $12(60.0)$ & 0.16 & $106(42.2)$ & $121(38.4)$ & 0.39 & $138(41.2)$ & 0.87 \\
\hline PH-2 & $0(0)$ & 1.00 & $12(4.7)$ & $12(3.8)$ & 0.58 & $13(3.9)$ & 0.61 \\
\hline Any PH & $1(5.0)$ & 1.00 & $20(7.9)$ & $25(7.9)$ & 1.00 & $30(9.0)$ & 0.77 \\
\hline Final infarct volume, $\mathrm{mL}$ & $\begin{array}{l}43.28 \\
{[22.94-85.17]}\end{array}$ & 0.81 & $\begin{array}{l}27.91 \\
{[9.66-55.11]}\end{array}$ & $\begin{array}{l}27.16 \\
{[11.69-67.27]}\end{array}$ & 0.35 & $\begin{array}{l}24.64 \\
{[8.77-61.74]}\end{array}$ & 0.55 \\
\hline 90-day good outcome & $5(29.4)$ & 0.31 & $105(44.5)$ & $125(43.7)$ & 0.86 & $147(46.4)$ & 0.67 \\
\hline 90-day mortality & $5(29.4)$ & 0.77 & $58(24.6)$ & $76(26.6)$ & 0.62 & $67(21.1)$ & 0.36 \\
\hline
\end{tabular}

IQR, interquartile range; mTICI, modified Thrombolysis in Cerebral Infarction; PH, parenchymal hematoma.

Table 3. Independent predictors of good outcomes (90-day mRS $0-2$ )

\begin{tabular}{lllc}
\hline & OR & $95 \%$ CI & $p$ value \\
\hline Age & 0.96 & $(0.94-0.97)$ & $<0.001$ \\
LDL & 1.01 & $(1.00-1.01)$ & 0.03 \\
Glucose & 0.99 & $(0.989-0.998)$ & 0.002 \\
INR & 0.62 & $(0.34-1.13)$ & 0.12 \\
SBP & 0.993 & $(0.986-1.000)$ & 0.06 \\
bNIHSS & 0.89 & $(0.85-0.92)$ & $<0.001$ \\
ASPECTS & 1.29 & $(1.29-1.48)$ & $<0.001$ \\
mTICI 2b-3 & 7.89 & $(3.21-19.39)$ & $<0.001$ \\
Any PH & 0.16 & $(0.06-0.39)$ & $<0.001$ \\
\hline
\end{tabular}

ASCPECTS, Alberta Stroke Program Early CT Score; bNIHSS, baseline National Institutes of Health Stroke Scale; CI, confidence interval; INR, international normalized ratio; LDL, low-density lipoprotein; mRS, modified Rankin scale; mTICI, modified Thrombolysis in Cerebral Infarction; OR, odds ratio; $\mathrm{PH}$, parenchymal hematoma; SBP, systolic blood pressure.

Counterintuitively, several studies have shown that BMI was associated with better outcomes after stroke [5-9]. This phenomenon is referred to as the "obesity stroke paradox." In a study published in 2008, Olsen et al. [5] showed that BMI was inversely related to mortality after stroke. All-cause mortality was lower in overweight (HR 0.73, 95\% CI 0.660.81 ), obese (HR 0.84, 95\% CI 0.73-0.98), and severely obese (HR 0.84, 95\% CI 0.64-1.10) patients than in normal weight patients. In a subsequent analysis of the same Danish registry, obese and overweight patients had a significantly lower risk of stroke recurrence and lower hospital readmission rates [6,7]. Similar findings were observed in other studies, such as in the Telemedical Project for Integrative Stroke Care (TEMPiS) trial [8]. On the other hand, other investigators showed results contradicting the latter with no improved outcomes in overweight or obese patients [10-14]. A recent meta-analysis including 25 studies and around 300,000 patients found that there was no obesity paradox in patients receiving intravenous thrombolysis [14]. The authors identified several methodological/design concerns that may 
have led some investigators to conclude that overweight and obesity were associated with better outcomes.

First, this could be explained by selection bias. Interestingly, the authors from the above referenced Danish registry, after further analysis of the same data, did not find an obesity paradox and attributed their earlier findings to selection bias [10]. In fact, patients who had more severe strokes were missing BMI data, which may have influenced the observed results. Second, time to follow-up in those studies varied greatly ranging from 1 week to 11.5 years, which could be too short to uncover any harmful effects of obesity on outcomes [14]. Third, these results could be explained by a confounding bias. In fact, as observed in our study, BMI is known to be inversely related to age, which is a well-established predictor of outcomes in stroke. Moreover, younger patients are less likely to have cardioembolic strokes and atrial fibrillation which have been shown to be associated with more severe strokes, large infarct volumes, and higher rates of hemorrhagic transformation and, therefore, carry a worse prognosis. Additionally, a recent analysis showed that both the severity and duration of obesity are important in the development of metabolic syndrome, meaning that "healthy" obesity is only a transitional state [16]. Our patients might have been in that transitional healthy state and developed a stroke as their first cardiovascular event, and, thus, a longer observation period beyond 90 days might help better assess outcomes in this population. Another potential explanation of this phenomenon could be related to a reverse causation bias, meaning severely ill patients often lose weight before death, leading to an underestimation of the risks of overweight and obesity. Yu et al. [17] found that this effect could be reversed when incorporating weight history in the analysis and that maximum BMI could be a potentially useful measure to minimize this bias. Another possible confounding parameter could be smoking, as both are risk factors of stroke but inversely correlated to each other. In our series, there were fewer smokers amongst obese patients. Smoking might lead to less fat but worse outcomes. Stokes and Preston [18] showed that when the analysis was restricted to never-smokers in patients with cardiovascular diseases, mortality risks were significantly higher in the overweight/ obesity group. Finally, a measurement bias could be the cause of the confusion (e.g., how accurate is the BMI in diagnosing obesity?). Romero-Corral et al. [19] found that a BMI cutoff of 30 had good specificity but missed $50 \%$ of patients with excess fat. Alternative measures, such as waist-to-hip ratio, waist circumference, or body composition (i.e., percentage of fat), might be more reliable [5, 20]. Last, in addition to statistical modeling/ distortion, a treatment bias may be another reason for the unexpected phenomenon. In an analysis of the Get With The Guidelines database, Steinberg et al. [21] found that physicians treat obese patients with coronary artery disease more aggressively due to assumed increase of vascular risk with better use of guideline-recommended medical treatment and invasive management.

Our study showed that despite differences in baseline characteristics, there were no differences in outcomes between BMI groups in patients undergoing mechanical thrombectomy. This held true after adjustment for potential confounders. Interestingly, underweight patients had numerically larger final infarct volumes, lower rates of good functional outcomes, and higher mortality rates. An association between underweight and stroke outcomes has been previously described, specifically the roles of underlying disease, body composition, physical fitness, and dietary intake, as they might be responsible for some or all of the apparent associations between underweight and mortality [2, 5]. Yu et al.'s [17] consideration of illness-induced weight loss in underweight patient outcomes suggests that collecting a more thorough weight history may prove informative.

One might ask why would there be a difference between patients undergoing mechanical thrombectomy. Catheterization in obese patients could be technically more 
challenging. Access to the femoral vessels may be difficult, and hemostasis might be more problematic to achieve. Moreover, visceral fat might contribute to aortic arch tortuosity, which could impact procedural times and outcomes [22]. Additionally, commercially available radiographic tables are unable to support the weight of more than $400 \mathrm{lbs}$. Another concern is treatment delays in transporting patients from a stretcher to CT and then back to the angiography suite given logistic reasons and the need for more personnel. This ultimately might lead to morbidly obese patients facing delays or not being offered treatment from which they could benefit. In our series, there were no differences between overweight/obese and normal weight patients and procedural length and outcomes. We believe that thrombectomy should be offered to eligible stroke patients regardless of their weight.

Our study has several limitations mostly inherent in its retrospective design and relatively moderate sample size. We did not have a control group that would have allowed us to measure treatment effect across BMI categories as well as access to treatment. Also, some patients were missing BMI data and were excluded from our study, which could have confounded our results. Furthermore, we only relied on BMI as the sole measure of obesity, which was the only measure consistently available for our patients on admission. Our results might have been different had we used other measures as referenced above. However, to the best of our knowledge, this is the first and largest series of patients undergoing stroke ET investigating the impact of weight on outcomes. This becomes specifically important given the increase in use of mechanical thrombectomy in stroke care and the increasing prevalence of obesity now affecting $35.5 \%$ of men and women in the US.

\section{Conclusions}

In stroke patients treated with mechanical thrombectomy, BMI does not seem to be associated with outcomes. However, patients who are overweight or obese have more comorbidities and higher stroke risk and, thus, should strive for a normal weight. Future larger prospective studies are warranted.

\section{Statement of Ethics}

This study was approved by the Emory University institutional review board.

\section{Disclosure Statement}

M.B., H.P., C.M.B., D.C.H., J.A.G., A.A., S.R.B., N.A.B., and M.R.F.: no disclosures.

R.G.N.: Stryker Neurovascular (Trevo-2 Trial PI, modest; DAWN Trial PI, no payment; Trevo Registry Steering Committee, no payment; Physician Advisory Board/Consultant, significant); Covidien/Medtronic (SWIFT and SWIFT-PRIME Steering Committee, modest; STAR Trial Core Lab, significant); and Penumbra (3-D Separator Trial Executive Committee, no payment); Neuravi/Cerenovus (ARISE-II Trial Steering Committee, no payment; Physician Advisory Board, modest); Phenox (Physician Advisory Board/ Consultant, modest); and Genentech (Physician Advisory Board/Consultant, modest).

\section{Funding Sources}

This research received no specific grant from any funding agency. 


\begin{tabular}{l|l}
\hline DOI: $10.1159 / 000496703$ & $\begin{array}{l}\text { C } 2019 \text { S. Karger AG, Basel } \\
\text { www.karger.com/ine }\end{array}$ \\
\hline
\end{tabular}

Bouslama et al.: Body Mass Index and Clinical Outcomes in Large Vessel Occlusion Acute Ischemic Stroke after Endovascular Therapy

\section{References}

1 Flegal KM, Carroll MD, Kit BK, Ogden CL. Prevalence of obesity and trends in the distribution of body mass index among US adults, 1999-2010. JAMA. 2012 Feb;307(5):491-7.

2 Flegal KM, Graubard BI, Williamson DF, Gail MH. Excess deaths associated with underweight, overweight, and obesity. JAMA. 2005 Apr;293(15):1861-7.

3 Flegal KM, Kit BK, Orpana H, Graubard BI. Association of all-cause mortality with overweight and obesity using standard body mass index categories: a systematic review and meta-analysis. JAMA. 2013 Jan;309(1):71-82.

4 Kernan WN, Inzucchi SE, Sawan C, Macko RF, Furie KL. Obesity: a stubbornly obvious target for stroke prevention. Stroke. 2013 Jan;44(1):278-86.

5 Olsen TS, Dehlendorff C, Petersen HG, Andersen KK. Body mass index and poststroke mortality. Neuroepidemiology. 2008;30(2):93-100.

6 Andersen KK, Olsen TS. Body mass index and stroke: overweight and obesity less often associated with stroke recurrence. J Stroke Cerebrovasc Dis. 2013 Nov;22(8):e576-81.

7 Andersen KK, Olsen TS. The obesity paradox in stroke: lower mortality and lower risk of readmission for recurrent stroke in obese stroke patients. Int J Stroke. 2015 Jan;10(1):99-104.

8 Doehner W, Schenkel J, Anker SD, Springer J, Audebert HJ. Overweight and obesity are associated with improved survival, functional outcome, and stroke recurrence after acute stroke or transient ischaemic attack: observations from the TEMPiS trial. Eur Heart J. 2013 Jan;34(4):268-77.

9 Vemmos K, Ntaios G, Spengos K, Savvari P, Vemmou A, Pappa T, et al. Association between obesity and mortality after acute first-ever stroke: the obesity-stroke paradox. Stroke. 2011 Jan;42(1):30-6.

10 Dehlendorff C, Andersen KK, Olsen TS. Body mass index and death by stroke: no obesity paradox. JAMA Neurol. 2014 Aug;71(8):978-84.

11 Branscheidt M, Schneider J, Michel P, Eskioglou E, Kaegi G, Stark R, et al. No Impact of Body Mass Index on Outcome in Stroke Patients Treated with IV Thrombolysis BMI and IV Thrombolysis Outcome. PLoS One. 2016 Oct;11(10):e0164413.

12 Ferreira I, Stehouwer CD. Obesity paradox or inappropriate study designs? Time for life-course epidemiology. J Hypertens. 2012 Dec;30(12):2271-5.

13 Sarikaya H, Elmas F, Arnold M, Georgiadis D, Baumgartner RW. Impact of obesity on stroke outcome after intravenous thrombolysis. Stroke. 2011 Aug;42(8):2330-2.

14 Oesch L, Tatlisumak T, Arnold M, Sarikaya H. Obesity paradox in stroke - Myth or reality? A systematic review. PLoS One. 2017 Mar;12(3):e0171334.

15 Strazzullo P, D’Elia L, Cairella G, Garbagnati F, Cappuccio FP, Scalfi L. Excess body weight and incidence of stroke: meta-analysis of prospective studies with 2 million participants. Stroke. 2010 May;41(5):e418-26.

16 Mongraw-Chaffin M, Foster MC, Kalyani RR, Vaidya D, Burke GL, Woodward M, et al. Obesity Severity and Duration Are Associated With Incident Metabolic Syndrome: Evidence Against Metabolically Healthy Obesity From the Multi-Ethnic Study of Atherosclerosis. J Clin Endocrinol Metab. 2016 Nov;101(11):4117-24.

17 Yu E, Ley SH, Manson JE, Willett W, Satija A, Hu FB, et al. Weight History and All-Cause and Cause-Specific Mortality in Three Prospective Cohort Studies. Ann Intern Med. 2017 May;166(9):613-20.

18 Stokes A, Preston SH. Smoking and reverse causation create an obesity paradox in cardiovascular disease. Obesity (Silver Spring). 2015 Dec;23(12):2485-90.

19 Romero-Corral A, Somers VK, Sierra-Johnson J, Thomas RJ, Collazo-Clavell ML, Korinek J, et al. Accuracy of body mass index in diagnosing obesity in the adult general population. Int J Obes. 2008 Jun;32(6):959-66.

20 De Lorenzo A, Soldati L, Sarlo F, Calvani M, Di Lorenzo N, Di Renzo L. New obesity classification criteria as a tool for bariatric surgery indication. World J Gastroenterol. 2016 Jan;22(2):681-703.

21 Steinberg BA, Cannon CP, Hernandez AF, Pan W, Peterson ED, Fonarow GC. Medical therapies and invasive treatments for coronary artery disease by body mass: the "obesity paradox" in the Get With The Guidelines database. Am J Cardiol. 2007 Nov;100(9):1331-5.

22 Mochida M, Sakamoto H, Sawada Y, Yokoyama H, Sato M, Sato H, et al. Visceral fat obesity contributes to the tortuosity of the thoracic aorta on chest radiograph in poststroke Japanese patients. Angiology. 2006 Jan-Feb; 57(1):85-91. 